\title{
Oscillation of Higher Order Linear Impulsive Dynamic Equations on Time Scales
}

\author{
Chaolong Zhang ${ }^{1,2}$, Feiqi Deng ${ }^{*}$ \\ ${ }^{1}$ College of Automation Science and Engineering, South China University of Technology, Guangzhou, China \\ ${ }^{2}$ Department of Computational Science, Zhongkai University Agriculture and Engineering, Guangzhou, China \\ Email: zhcl88@126.com, *aufqdeng@scut.edu.cn
}

Received February 18, 2012; revised April 17, 2012; accepted April 24, 2012

\begin{abstract}
In this paper, we will establish some oscillation criteria for the higher order linear dynamic equation on time scale in term of the coefficients and the graininess function. We illustrate our results with an example.
\end{abstract}

Keywords: Oscillation; High Order; Dynamic Equation; Time Scale; Riccati Tramsformation

\section{Introduction}

Since Stefen Hilger formed the definition of derivatives and integrals on time scales, several authors has expounded on various aspects of the new theory, see the papers by Agarwal et al. [1] and the references cited therein.

A book on the subject of time scale, i.e., measure chain, by Bohner and Peterson [2] summarize and organizes much of time scale calculus on time scale and references given therein.

A time scale $\mathbb{T}$ is an arbitrary closed subset reals, and the cases when this tie sale is equal to the reals or to the integers represent the classical theories of differential and of difference equations.

In recent years there has been much research activity concerning the oscillation and non-oscillation of solution of some differential equations on time scales,we refer the reader to the few papers [3-7].

In [4], the authors considered the second order dynamic equation

$$
\left(p(t) x^{\Delta}(t)\right)^{\Delta}+q(t) x(\sigma(t))=0,
$$

and some sufficient conditions for oscillation of all solution on unbounded time scales are given. But, the oscillation criteria are not considered the impulsive influence. It is rarely about the oscillation of higher order impulsive dynamic equations on time scales.

In this paper we shall consider the following linear higher order impulsive dynamic equation

${ }^{*}$ Corresponding author.

$$
\left\{\begin{array}{l}
x^{\Delta^{n}}+p(t) x^{\sigma}(t)=0, t \in \mathbb{J}_{\mathbb{T}}:=[0, \infty) \cap \mathbb{T}, \\
\quad t \neq t_{k}, k=1,2, \cdots, \\
x^{\Delta^{i}}\left(t_{k}^{+}\right)=a_{k}^{(i)} x^{\Delta^{i}}\left(t_{k}\right), i=0,1, \cdots, n-1, k=1,2 \cdots \\
x\left(t_{0}^{+}\right)=x_{0}, x^{\Delta^{i}}\left(t_{0}^{+}\right)=x_{0}^{\Delta^{i}} .
\end{array}\right.
$$

where $n$ is even, $a_{k}^{(i)}>0, p(t)$ is positive real-valued rd-continuous functions defined on the time scales and

$$
\begin{aligned}
& \left(H_{1}\right): \int_{t_{0}}^{+\infty} \prod_{t_{0}<t_{k}<s} \frac{a_{k}^{(i)}}{a_{k}^{(i-1)}} \Delta s=+\infty . \\
& f^{\Delta^{i}}(t)=\lim _{s \rightarrow t} \frac{f^{\Delta^{i-1}}(\sigma(t))-f^{\Delta^{i-1}}(s)}{\sigma(t)-s}, s \in \mathbb{T}\{\sigma(t)\} . \\
& x\left(t_{k}^{+}\right)=\lim _{h \rightarrow 0^{+}} x\left(t_{k}+h\right), \quad x^{\Delta^{i}}\left(t_{k}^{+}\right)=\lim _{h \rightarrow 0^{+}} x^{\Delta^{i}}\left(t_{k}+h\right),
\end{aligned}
$$

Throughout the remainder of the paper, we assume that, for each $k=1,2, \cdots$, the points of impulses $t_{k}$ are right dense (rd for short). In order to define the solutions of the problem (1), we introduce the following space

$$
\begin{aligned}
A C^{i}= & \left\{x: \mathbb{J}_{\mathbb{T}} \rightarrow \mathbb{R} \text { is } i \text {-times } \Delta\right. \text {-differentiable, whose } \\
& \left.i \text {-th delta-derivative } x^{\Delta^{i}} \text { is absolutely continuous }\right\} . \\
P C= & \left\{x: \mathbb{J}_{\mathbb{T}} \rightarrow \mathbb{R}\right. \text { is rd-continuous expect at the points } \\
& t_{k}, k=1,2, \cdots, \text { for which } y\left(t_{k}^{-}\right), x\left(t_{k}^{+}\right), x^{\Delta^{i}}\left(t_{k}^{-}\right) \text {and } \\
& \left.x^{\Delta}\left(t_{k}^{+}\right) \text {exist with } x\left(t_{k}^{-}\right)=x\left(t_{k}\right), x^{\Delta^{i}}\left(t_{k}^{-}\right)=x^{\Delta^{i}}\left(t_{k}\right)\right\} .
\end{aligned}
$$


Definition 1. A function $y \in P C \cap A C^{i}\left(\mathbb{J}_{\mathbb{T}} \backslash\left\{t_{1}, \cdots\right\}, \mathbb{R}\right)$ is said to be a solution of (1), if it satisfies

$x^{\Delta^{n}}(t)+p(t) x^{\sigma}(t)=0$ a.e. on $\mathbb{J}_{\mathbb{T}} \backslash\left\{t_{k}\right\}, k=1,2, \cdots$, and for each $k=1,2, \cdots, x$ satisfies the impulsive condition $x^{\Delta^{i}}\left(t_{k}^{+}\right)=a_{k}^{(i)} x^{\Delta^{i}}\left(t_{k}\right)$ and the initial conditions $x\left(t_{0}^{+}\right)=x_{0}$, $x^{\Delta^{i}}\left(t_{0}^{+}\right)=x_{0}^{\Delta^{i}}$.

Before doing so, let us first recall that a solution of (1) is a nontrivial real function $x(t)$ satisfying Equation (1) for $t \geq a$. A solution $x(t)$ of (1) is said to be oscillatory if it is neither eventually positive nor eventually negative, otherwise it is non-oscillatory. Equation (1) is said to be oscillatory if all its solutions are oscillatory. Our attention is restricted to those solutions of (1) which exist on some half line $\left[t_{x}, \infty\right)$ and satisfy

$\sup \left\{|x(t)|: t>t_{0}\right\}>0$ for any $t \geq t_{x}$.

\section{Preliminaries}

A time scale $\mathbb{T}$ is an arbitrary non-empty closed subset of the real numbers $\mathbb{R}$. Since we are interested in oscillatory behavior, we suppose that the time scale under consideration is not bounded above, i.e., it is a time scale interval of the form $[a, \infty)$. On any time scale we define the forward and backward jump operators by

$$
\sigma(t):=\inf \{s \in \mathbb{T}: s>t\}, \rho(t):=\sup \{s \in \mathbb{T}: s<t\} .
$$

A point $t$ is said to be left-dense if $\rho(t)=t$, right-dense if $\sigma(t)=t$, left scattered if $\rho(t)<t$, and right-scattered if $\sigma(t)>t$. The graininess $\mu$ of the time scale is define by $\mu(t)=\sigma(t)-t$. The set $\mathbb{T}^{k}$ is derived from $\mathbb{T}$ as follow: If $\mathbb{T}$ has a left-scattered maximum $m$, then $\mathbb{T}^{k}=\mathbb{T}-\{m\}$; otherwise, $\mathbb{T}^{k}=\mathbb{T}$.

For a function $f: \mathbb{T} \rightarrow \mathbb{R}$ (the range $\mathbb{R}$ of may actually be replaced by any Banach space), the (delta) derivative is defined by

$$
f^{\Delta}(t)=\lim _{s \rightarrow t} \frac{f(\sigma(t))-f(s)}{\sigma(t)-s}, s \in \mathbb{T}\{\sigma(t)\} .
$$

A function $f:[a, b] \rightarrow \mathbb{R}$ is said to be re-continuous at each right-dense point and if there exists a finite left limit in all left-dense points, and $f$ is said to be differentiable if its derivative exists, the derivative and the shift operator $\sigma$ are related by the formula

$$
f^{\sigma}=f+\mu f^{\Delta} \text {, where } f^{\sigma}:=f \circ \sigma .
$$

We will make use of the following product and quotient rules for the derivative of the product $f g$ and the quotient $f / g$ of two differentiable functions $f$ and $g$

$$
\begin{aligned}
(f g)^{\Delta} & =f^{\Delta}(t) g(t)+f(\sigma(t)) g^{\Delta}(t) \\
& =f(t) g^{\Delta}(t)+f^{\Delta}(t) g(\sigma(t)),
\end{aligned}
$$

$$
\left(\frac{f}{g}\right)^{\Delta}(t)=\frac{f^{\Delta}(t) g(t)-f(t) g^{\Delta}(t)}{g(t) g(\sigma(t))} .
$$

The integration by parts formula reads

$$
\begin{aligned}
\int_{a}^{b} f^{\Delta}(t) g(t) \Delta t= & f(b) g(b)-f(a) g(a) \\
& -\int_{a}^{b} f^{\sigma}(t) g^{\Delta}(t) \Delta t .
\end{aligned}
$$

Remark 1. We note that if $\mathbb{T}=\mathbb{R}$, then $\sigma(t)=t$, $\mu(t)=0, f^{\Delta}(t)=f^{\prime}(t)$ and (1) becomes the higher order differential equation

$$
\left\{\begin{array}{l}
x^{(n)}(t)+p(t) x(t)=0, t \neq t_{k}, k=1,2, \cdots, \\
x^{(i)}\left(t_{k}^{+}\right)=a_{k}^{(i)} x^{(i)}\left(t_{k}\right), i=0,1, \cdots, n-1, k=1,2 \cdots \\
x\left(t_{0}^{+}\right)=x_{0}, x^{(i)}\left(t_{0}^{+}\right)=x_{0}^{(i)} .
\end{array}\right.
$$

If $\mathbb{T}=\mathbb{Z}$, then $\sigma(t)=t+1, \mu(t)=1$,

$$
x^{\Delta^{n}}(t)=\Delta^{n} x(t)=\Delta^{n-1} x(t+1)-\Delta^{n-1} x(t),
$$

and (1) becomes the higher order difference equation

$$
\left\{\begin{array}{l}
\Delta^{n} x(t)+p(t) x(t+1)=0, t \neq t_{k}, k=1,2, \cdots, \\
\Delta^{i} x\left(t_{k}^{+}\right)=a_{k}^{(i)} \Delta^{i} x\left(t_{k}\right), i=0,1, \cdots, n-1, k=1,2 \cdots \\
x\left(t_{0}^{+}\right)=x_{0}, \Delta^{i} x\left(t_{0}^{+}\right)=\Delta^{i} x_{0} .
\end{array}\right.
$$

If $\mathbb{T}=h \mathbb{Z}, h>0$, then $\sigma(t)=t+h, \mu(t)=h$,

$$
x^{\Delta}(t)=\Delta x_{h}(t)=\frac{x(t+h)-x(t)}{h},
$$

and (1) becomes the higher order difference equation

$$
\left\{\begin{array}{l}
\Delta_{h}^{n} x(t)+p(t) x(t+h)=0, t \neq t_{k}, k=1,2, \cdots, \\
\Delta_{h}^{i} x\left(t_{k}^{+}\right)=a_{k}^{(i)} \Delta_{h}^{i} x\left(t_{k}\right), i=0,1, \cdots, n-1, k=1,2 \cdots \\
x\left(t_{0}^{+}\right)=x_{0}, \Delta_{h}^{i} x\left(t_{0}^{+}\right)=\Delta_{h}^{i} x_{0} .
\end{array}\right.
$$

If $\mathbb{T}=q^{\mathbb{N}}=\left\{t: t=q^{k}, k \in \mathbb{N}, q>1\right\}$, then $\sigma(t)=q t$, $\mu(t)=(q-1) t$,

$$
x^{\Delta} t=\Delta_{q} x(t)=\frac{x(q t)-x(t)}{(q-1) t}
$$

and (1) becomes the higher order difference equation

$$
\left\{\begin{array}{l}
\Delta_{q}^{n} x(t)+p(t) x(q t)=0, t \neq t_{k}, k=1,2, \cdots, \\
\Delta_{q}^{i} x\left(t_{k}^{+}\right)=a_{k}^{(i)} \Delta_{q}^{i} x\left(t_{k}\right), i=0,1, \cdots, n-1, k=1,2 \cdots \\
x\left(t_{0}^{+}\right)=x_{0}, \Delta_{q}^{i} x\left(t_{0}^{+}\right)=\Delta_{q}^{i} x_{0} .
\end{array}\right.
$$

If $\mathbb{N}_{0}^{2}=\left\{t^{2}: t \in \mathbb{N}_{0}\right\}$, then $\sigma(t)=(\sqrt{t}+1)^{2}$ and 
$\mu(t)=1+2 \sqrt{t}$,

$$
\Delta x_{N}(t)=\frac{x\left((\sqrt{t}+1)^{2}\right)-x(t)}{1+2 \sqrt{t}}
$$

and (1) becomes the higher order difference equation

$$
\left\{\begin{array}{l}
\Delta_{N}^{n} x(t)+p(t) x(1+2 \sqrt{t}+t)=0, t \neq t_{k}, k=1,2, \cdots, \\
\Delta_{N}^{i} x\left(t_{k}^{+}\right)=a_{k}^{(i)} \Delta_{N}^{i} x\left(t_{k}\right), i=0,1, \cdots, n-1, k=1,2 \cdots \\
x\left(t_{0}^{+}\right)=x_{0}, \Delta_{N}^{i} x\left(t_{0}^{+}\right)=\Delta_{N}^{i} x_{0} .
\end{array}\right.
$$

\section{Main Results}

In the following, we will prove some lemmas, which will be useful for establishing oscillation criteria .

Lemma 1. Let $y, f \in C_{r d}$ and $p \in \mathcal{R}^{+}$. Then

$$
y^{\Delta}(t) \leq p(t) y(t)+f(t)
$$

implies, for all $t \in \mathbb{T}$

$$
y(t) \leq y\left(t_{0}\right) e_{p}\left(t, t_{0}\right)+\int_{t_{0}}^{t} e_{p}(l, \sigma(s)) f(s) \Delta s
$$

See $\left[2, P_{255}\right]$.

Lemma 2. Assume that $m \in P C^{1}[\mathbb{T}, \mathbb{R}]$ and

$$
\begin{aligned}
& m^{\Delta}(t) \leq p(t) m(t)+q(t), \\
& \quad t \in \mathbb{J}_{\mathbb{T}}:=[0, \infty) \cap \mathbb{T}, t \neq t_{k}, k=1,2, \cdots, \\
& m\left(t_{k}^{+}\right) \leq d_{k} m\left(t_{k}^{-}\right)+b_{k}, k=1,2, \cdots,
\end{aligned}
$$

then for $t \geq t_{0}$

$$
\begin{aligned}
m(t) \leq & m\left(t_{0}\right) \prod_{t_{0}<t_{k}<t} \mathrm{~d}_{k} e_{p}\left(t, t_{0}\right) \\
& +\sum_{t_{0}<t_{k}<t}\left(\prod_{t_{k}<t_{j}<t} \mathrm{~d}_{j} e_{p}\left(t, t_{k}\right)\right) b_{k} \\
& +\int_{t_{0}}^{t} \prod_{s<t_{k}<t} \mathrm{~d}_{k} e_{p}(t, \sigma(s)) q(s) \Delta s .
\end{aligned}
$$

Proof. Let $t \in\left[t_{0}, t_{1}\right]_{\mathbb{T}}$, use Lemma 1 , we obtain

$$
\begin{gathered}
m(t) \leq m\left(t_{0}\right) e_{p}\left(t, t_{0}\right)+\int_{t_{0}}^{t} e_{p}(t, \sigma(s)) q(s) \Delta s \\
t \in\left[t_{0}, t_{1}\right]_{\mathbb{T}} .
\end{gathered}
$$

Hence (10) is true for $t \in\left[t_{0}, t_{1}\right]_{\mathbb{T}}$. Now assume that (10) holds for $t \in\left[t_{0}, t_{n}\right]_{\mathbb{T}}$ for some integer $n>1$. Then for $t \in\left(t_{n}, t_{n+1}\right]_{\mathbb{T}}$, it follows from (9) and Lemma 1, we get

$$
m(t) \leq m\left(t_{n}^{+}\right) e_{p}\left(t, t_{n}\right)+\int_{t_{n}}^{t} e_{p}(t, \sigma(s)) q(s) \Delta s
$$

Using (9), we obtain from (10)

$$
\begin{aligned}
m(t) \leq & {\left[\mathrm{d}_{n} m\left(t_{n}^{-}\right)+b_{n}\right] e_{p}\left(t, t_{n}\right)+\int_{t_{n}}^{t} e_{p}(t, \sigma(s)) q(s) \Delta s } \\
\leq & \mathrm{d}_{n} e_{p}\left(t, t_{n}\right)\left[m\left(t_{0}\right) \prod_{t_{0}<t_{k}<t_{n}} \mathrm{~d}_{k} e_{p}\left(t_{n}, t_{0}\right)\right. \\
& +\sum_{t_{0}<t_{k}<t_{n}}\left(\prod_{t_{k}<t_{j}<t_{n}} \mathrm{~d}_{j} e_{p}\left(t_{n}, t_{k}\right)\right) b_{k} \\
& \left.+\int_{t_{0}}^{t_{n}} \prod_{s<t_{k}<t_{n}} \mathrm{~d}_{k} e_{p}\left(t_{n}, \sigma(s)\right) q(s) \Delta s\right] \\
& +b_{n} e_{p}\left(t, t_{n}\right)+\int_{t_{n}}^{t} e_{p}(t, \sigma(s)) q(s) \Delta s \\
\leq & m\left(t_{0}\right) \prod_{t_{0}<t_{k}<t} \mathrm{~d}_{k} e_{p}\left(t, t_{0}\right) \\
& +\sum_{t_{0}<t_{k}<t}\left(\prod_{t_{k}<t_{j}<t} \mathrm{~d}_{j} e_{p}\left(t, t_{k}\right)\right) b_{k} \\
& +\int_{t_{0}}^{t} \prod_{s<t_{k}<t} \mathrm{~d}_{k} e_{p}(t, \sigma(s)) q(s) \Delta s,
\end{aligned}
$$

which on simplification gives the estimate (10) for $t \in\left[t_{0}, t_{n+1}\right]_{\mathbb{T}}$, by induction, we get (10) holds for $t \geq t_{0}$.

Lemma 3. Let $x(t)$ be a solution of (1), and conditions $\left(H_{1}\right)$ are satisfied. Suppose that there exists an $i \in\{1,2, \cdots, n-1\}$ and some $T \geq t_{0}$, such that $x^{\Delta^{i+1}}(t) \geq 0(\leq 0), x^{\Delta^{i}}(t)>0(<0)$ for $t \geq T$. Then, there exists some $T_{1} \geq T$ such that $x^{\Delta^{i-1}}(t)>0(<0)$ for $t \geq T_{1}$.

Proof. Without loss of generality, let $T=t_{0}$. Assume that for any $t_{k}>T, x^{\Delta^{i-1}}\left(t_{k}\right)<0$. By $x^{\Delta^{i+1}}(t) \geq 0$, $x^{\Delta^{i}}(t)>0, t \in\left(t_{k}, t_{k+1}\right]_{\mathbb{T}}$, we have that $x^{\Delta^{i}}(t)$ is monotonically nondecreasing on $\left(t_{k}, t_{k+1}\right]_{\mathbb{T}}$. For $t \in\left(t_{1}, t_{2}\right]_{\mathbb{T}}$, we have

$$
x^{\Delta^{i}}(t) \geq x^{\Delta^{i}}\left(t_{1}^{+}\right)
$$

Integrating the above inequality, we have

$$
x^{\Delta^{i-1}}\left(t_{2}\right) \geq x^{\Delta^{i-1}}\left(t_{1}^{+}\right)+x^{\Delta^{i}}\left(t_{1}^{+}\right) \int_{t_{1}}^{t_{2}} \Delta t
$$

Similar to (11),

$$
x^{\Delta^{i-1}}\left(t_{3}\right) \geq x^{\Delta^{i-1}}\left(t_{2}^{+}\right)+x^{\Delta^{i}}\left(t_{2}^{+}\right) \int_{t_{2}}^{t_{3}} \Delta t .
$$

By $x^{\Delta^{i}}\left(t_{2}\right) \geq x^{\Delta^{i}}\left(t_{1}^{+}\right)$and (11), (12), we have

$$
\begin{aligned}
& x^{\Delta^{i-1}}\left(t_{3}\right) \geq x^{\Delta^{i-1}}\left(t_{2}^{+}\right)+x^{\Delta^{i}}\left(t_{2}^{+}\right) \int_{t_{2}}^{t_{3}} \Delta t \\
& =a_{2}^{(i-1)} x^{\Delta^{i-1}}\left(t_{2}\right)+a_{2}^{(i)} x^{\Delta^{i}}\left(t_{2}\right) \int_{t_{2}}^{t_{3}} \Delta t \\
& =a_{2}^{(i-1)}\left[x^{\Delta^{i-1}}\left(t_{1}^{+}\right)+x^{\Delta^{i}}\left(t_{1}^{+}\right) \int_{t_{1}}^{t_{2}} \Delta t\right]+a_{2}^{(i)} x^{\Delta^{i}}\left(t_{2}\right) \int_{t_{2}}^{t_{3}} \Delta t \\
& =a_{2}^{(i-1)}\left[x^{\Delta^{i-1}}\left(t_{1}^{+}\right)+x^{\Delta^{i}}\left(t_{1}^{+}\right) \int_{t_{1}}^{t_{2}} \Delta t+\frac{a_{2}^{(i)}}{a_{2}^{(i-1)}} x^{\Delta^{i}}\left(t_{1}^{+}\right) \int_{t_{2}}^{t_{3}} \Delta t\right] .
\end{aligned}
$$

Applying induction we have, for any natural number $m$, 


$$
\begin{aligned}
& x^{\Delta^{i-1}}\left(t_{m}\right)=a_{m-1}^{(i-1)} \cdots a_{3}^{(i-1)} a_{2}^{(i-1)}\left\{x^{\Delta^{i-1}}\left(t_{1}^{+}\right)\right. \\
& +x^{\Delta^{i}}\left(t_{1}^{+}\right)\left[\int_{t_{1}}^{t_{2}} \Delta t+\frac{a_{2}^{(i)}}{a_{2}^{(i-1)}} \int_{t_{2}}^{t_{3}} \Delta t+\cdots\right. \\
& \left.\left.+\frac{a_{2}^{(i)} a_{3}^{(i)} \cdots a_{m-1}^{(i)}}{a_{2}^{(i-1)} a_{3}^{(i-1)} \cdots a_{m-1}^{(i-1)}} \int_{t_{m-1}}^{t_{m}} \Delta t\right]\right\} \\
& =a_{m-1}^{(i-1)} \cdots a_{3}^{(i-1)} a_{2}^{(i-1)}\left\{x^{\Delta^{i-1}}\left(t_{1}^{+}\right)\right. \\
& \left.+x^{\Delta^{i}}\left(t_{1}^{+}\right) \int_{t_{1}}^{t_{m}} \prod_{t_{1}<t_{k}<t_{m}} \frac{a_{k}^{(i)}}{a_{k}^{(i-1)}} \Delta t\right\}
\end{aligned}
$$

By condition $\left(H_{1}\right)_{i-1}$ and $a_{k}^{(i)}>0$, for all sufficiently large $m$, we have $x^{\Delta^{i-1}}\left(t_{m}\right)>0$. i.e., there exists a natural number $N$, when $m \geq N$, we have $x^{\Delta^{i-1}}\left(t_{m}\right)>0$. By $x^{\Delta^{i}}(t)>0$ again, we have $x^{\Delta^{i-1}}(t)>x^{\Delta^{i-1}}\left(t_{k}\right)>0$, for $t \in\left(t_{k}, t_{k+1}\right]_{\mathbb{T}}$. When $t \geq T_{1}$, we have $x^{\Delta^{i-1}}(t)>0$, where $t_{N}=T_{1}$. The proof of Lemma 3 is completed.

Lemma 4. Let $x(t)$ be a solution of (1) and conditions $\left(H_{1}\right)$ are satisfied. Suppose that there exists an $i \in\{1,2, \cdots, n\}$ and some $T \geq t_{0}$ such that $x(t)>0$, $x^{\Delta^{i}}(t) \leq 0$ for $t \geq T . x^{\Delta^{i}}(s)$ is not always equal to 0 in $[t,+\infty)_{\mathbb{T}}$ for $t \geq T$. Then we have $x^{\Delta^{1-1}}(t)>0$ for all sufficiently large $t$.

Proof. Without loss of generality, let $T=t_{0}$. We claim that $x^{\Delta^{i-1}}\left(t_{k}\right)>0$ for any $t_{k} \geq T$.

If it is not true, then there exists some $t_{j} \geq T$ such that $x^{\Delta^{i-1}}\left(t_{j}\right) \leq 0$. Since $x^{\Delta^{i}}(t) \leq 0, x^{\Delta^{i-1}}(t)$ is monotonically non-increasing in $\left(t_{k}, t_{k+1}\right]_{\mathbb{T}}$ for $k \geq j$. And because $x^{\Delta^{i}}(s)$ is not always equal to 0 in $[t,+\infty)_{\mathbb{T}}$, there exists some $t_{l} \geq t_{j}$ such that $x^{\Delta^{i}}(t)$ is not always equal to 0 in $\left(t_{l}, t_{l+1}\right]_{\mathbb{T}}$. Without loss of generality, we can assume $l=j$, that is, $x^{\Delta^{i}}(t)$ is not always equal to 0 in $\left(t_{j}, t_{j+1}\right]_{\mathbb{T}}$. So we have

$$
x^{\Delta^{i-1}}\left(t_{j+1}\right)<x^{\Delta^{i-1}}\left(t_{j}^{+}\right)=a_{j}^{(i-1)} x^{\Delta^{i-1}}\left(t_{j}\right) \leq 0 .
$$

For $t \in\left(t_{j+1}, t_{j+2}\right]_{\mathbb{T}}$, we have

$$
x^{\Delta^{i-1}}\left(t_{j+2}\right)<x^{\Delta^{i-1}}\left(t_{j+1}^{+}\right)=a_{j+1}^{(i-1)} x^{\Delta^{i-1}}\left(t_{j+1}\right)<0 .
$$

By induction, for $t \in\left(t_{j+m}, t_{j+m+1}\right]_{\mathbb{T}}$, we have $x^{\Delta^{i-1}}(t)<0$. So we have

$$
x^{\Delta^{i-1}}(t)<0, x^{\Delta^{i}}(t) \leq 0, t \in\left(t_{j+1},+\infty\right)_{\mathbb{T}} .
$$

By Lemma 3, for all sufficiently large $t$, we have $x^{\Delta^{i-2}}(t)<0$. Similarly, we can conclude, by using Lemma 3 repeatedly, that for all sufficiently large $t, x(t)<0$. This is a contradiction with $x(t)>0(t \geq T)$ ! Hence, we have $x^{\Delta^{i-1}}\left(t_{k}\right)>0$ for any $t_{k} \geq T$. So we have $x^{\Delta^{i-1}}(t)>0$ for all sufficiently large $t$. The proof of Lemma 4 is com- pleted.

Lemma 5. Let $x(t)$ be a solution of (1) and conditions $\left(H_{1}\right)$ are satisfied. Suppose $T \geq t_{0}$ and $x(t)>0$ for $t \geq T$. Then there exist some $T^{\prime} \geq T$ and $l \in\{1,3, \cdots, n-1\}$ such that for $t \geq T^{\prime}$,

$$
\left\{\begin{array}{l}
x^{\Delta^{i}}(t)>0, i=0,1, \cdots, l \\
(-1)^{i-1} x^{\Delta^{i}}(t)>0, i=l+1, \cdots, n-1 ; \\
x^{\Delta^{n}}(t) \leq 0 .
\end{array}\right.
$$

Proof. Let $T=t_{0}$, for $x(t)>0\left(t \geq t_{0}\right)$, by (1) and $p(t)$ is nonnegative and is not always equal to 0 in any $(t,+\infty)_{\mathbb{T}}$,

$$
x^{\Delta^{n}}(t)=-p(t) x^{\sigma}(t) \leq 0 .
$$

$x^{\Delta^{n}}(t)$ is not always equal to 0 in $(t,+\infty)_{\mathbb{T}}$, by Lemma 4. So we have $x^{\Delta^{n-1}}(t)<0$ for all sufficiently large $t$. Without loss of generality, let $t \geq t_{0}, x^{\Delta^{n-1}}(t)>0$. So $x^{\Delta^{n-2}}(t)$ is monotonically non-decreasing in $\left(t_{k}, t_{k+1}\right]_{\mathbb{T}}$. If for any $t_{k}, x^{\Delta^{n-2}}\left(t_{k}\right)<0$, then $x^{\Delta^{n-2}}(t)<0\left(t \geq t_{0}\right)$. If there exists some $t_{j}, x^{\Delta^{n-2}}\left(t_{j}\right) \geq 0$, by $x^{\Delta^{n-2}}(t)$ is monotonically nondecreasing and $a_{k}^{(n-2)}>0$, then $x^{\Delta^{n-2}}(t)>0$ for $t>t_{j}$. So there exists some $T_{1} \geq T$, when $t \geq T_{1}$, then one of the following statements holds:

$$
\begin{aligned}
& \left(A_{1}\right) \quad x^{\Delta^{n-1}}(t)>0, x^{\Delta^{n-2}}(t)>0, t \geq T_{1} ; \\
& \left(B_{1}\right) x^{\Delta^{n-1}}(t)>0, x^{\Delta^{n-2}}(t)<0, t \geq T_{1} ;
\end{aligned}
$$

when $\left(A_{1}\right)$ holds, by Lemma 3 , then $x^{\Delta^{n-3}}(t)>0$, for all sufficiently large $t$. By Lemma 3 over and over again, at last, for all sufficiently large $t$, we have

$$
x^{\Delta^{n-1}}(t)>0, x^{\Delta^{n-2}}(t)>0, \cdots, x^{\Delta}(t)>0, x(t)>0 .
$$

When $\left(B_{1}\right)$ holds, by Lemma 4 , then $x^{\Delta^{n-3}}(t)>0$, for all sufficiently large $t$. By deducing further, there exists some $T_{2} \geq T_{1}$, when $t \geq T_{2}$, then one of the following statements holds:

$$
\begin{aligned}
& \left(A_{2}\right) \quad x^{\Delta^{n-3}}(t)>0, x^{\Delta^{n-4}}(t)>0, t \geq T_{2} ; \\
& \left(B_{2}\right) x^{\Delta^{n-3}}(t)>0, x^{\Delta^{n-4}}(t)<0, t \geq T_{2} ;
\end{aligned}
$$

discuss the above over and over,eventually, there exists some $T^{\prime} \geq T$ and $l \in\{1,3, \cdots, n-1\}$, when $t \geq T^{\prime}$, we have

$$
\left\{\begin{array}{l}
x^{\Delta^{i}}(t)>0, i=0,1, \cdots, l . \\
(-1)^{i-1} x^{\Delta^{i}}(t)>0, i=l+1, l+2, \cdots, n-1 . \\
x^{\Delta^{n}}(t) \leq 0
\end{array}\right.
$$

The proof of Lemma 5 is completed.

Remark 2. If $x(t)$ is an eventually negative solution of (1),we have conclusions similar to Lemma 4 and 
Lemma 5.

Theorem 1. If conditions $\left(H_{1}\right)$ hold, and

$$
\int_{t_{0}}^{+\infty} \prod_{t_{0}<t_{k}<t} \frac{1}{a_{k}^{(n-1)}} p(t) \Delta t=+\infty .
$$

then every solution of (1) is oscillatory.

Proof. Let $x(t)$ be a non-oscillatory solution of (1). Without loss of generality, let $x(t)>0\left(t \geq t_{0}\right)$. By Lemma 5 and (1), there exists $T^{\prime} \geq t_{0}$, when $t \geq T^{\prime}$, we have

$$
x^{\Delta^{n}}(t) \leq 0, x^{\Delta^{n-1}}(t)>0, x^{\Delta}(t)>0, x(t)>0 .
$$

Let $T^{\prime}=t_{0}$. when $t_{k}>t_{0}, \quad x^{\Delta^{n-1}}(t)$ is monotonically non-increasing in $\left(t_{k}, t_{k+1}\right]_{\mathbb{T}}$ and $x(t)$ is monotonically increasing in $\left(t_{k}, t_{k+1}\right]_{\mathbb{T}}$.

By (1), we have

$$
x^{\Delta^{n}}(t)=-p(t) x^{\sigma}(t)
$$

Integrating (17) from $t_{0}$ to $t_{1}$ we have

$$
x^{\Delta^{n-1}}\left(t_{1}\right)-x^{\Delta^{n-1}}\left(t_{0}^{+}\right)=-\int_{t_{0}}^{t_{1}} p(t) x^{\sigma}(t) \Delta t,
$$

by the above equation and $x(t)$ is monotonically increasing, we have

$$
\begin{aligned}
x^{\Delta^{n-1}}\left(t_{1}\right) & =x^{\Delta^{n-1}}\left(t_{0}^{+}\right)-\int_{t_{0}}^{t_{1}} p(t) x^{\sigma}(t) \Delta t \\
& \leq x^{\Delta^{n-1}}\left(t_{0}^{+}\right)-x^{\sigma}\left(t_{0}^{+}\right) \int_{t_{0}}^{t_{1}} p(t) \Delta t,
\end{aligned}
$$

then

$$
x^{\Delta^{n-1}}\left(t_{1}\right) \leq x^{\Delta^{n-1}}\left(t_{0}^{+}\right)-x^{\sigma}\left(t_{0}^{+}\right) \int_{t_{0}}^{t_{1}} p(t) \Delta t,
$$

similar to (19), we have

$$
x^{\Delta^{n-1}}\left(t_{2}\right)=x^{\Delta^{n-1}}\left(t_{1}^{+}\right)-x^{\sigma}\left(t_{1}^{+}\right) \int_{t_{1}}^{t_{2}} p(t) \Delta t .
$$

By (19), (20) and $x(t)$ being monotonically increasing,

$$
\begin{aligned}
& x^{\Delta^{n-1}}\left(t_{2}\right) \leq x^{\Delta^{n-1}}\left(t_{1}^{+}\right)-x^{\sigma}\left(t_{1}^{+}\right) \int_{t_{1}}^{t_{2}} p(t) \Delta t \\
& =a_{1}^{(n-1)} x^{\Delta^{n-1}}\left(t_{1}\right)-x^{\sigma}\left(t_{0}^{+}\right) \int_{t_{1}}^{t_{2}} p(t) \Delta t \\
& \leq a_{1}^{(n-1)}\left[x^{\Delta^{n-1}}\left(t_{0}^{+}\right)-x^{\sigma}\left(t_{0}^{+}\right) \int_{t_{0}}^{t_{1}} p(t) \Delta t\right]-x^{\sigma}\left(t_{0}^{+}\right) \int_{t_{1}}^{t_{2}} p(t) \Delta t \\
& \leq a_{1}^{(n-1)}\left[x^{\Delta^{n-1}}\left(t_{0}^{+}\right)-x^{\sigma}\left(t_{0}^{+}\right) \int_{t_{0}}^{t_{1}} p(t) \Delta t-\frac{x^{\sigma}\left(t_{0}^{+}\right)}{a_{1}^{(n-1)}} \int_{t_{1}}^{t_{2}} p(t) \Delta t\right],
\end{aligned}
$$

similarly, we have

$$
x^{\Delta^{n-1}}\left(t_{3}\right) \leq x^{\Delta^{n-1}}\left(t_{2}^{+}\right)-x^{\sigma}\left(t_{2}^{+}\right) \int_{t_{2}}^{t_{3}} p(t) \Delta t
$$

then

$$
\begin{aligned}
x^{n^{n-1}}\left(t_{3}\right) \leq & a_{2}^{(n-1)} a_{1}^{(n-1)}\left[x^{\Delta^{n-1}}\left(t_{0}^{+}\right)-x^{\sigma}\left(t_{0}^{+}\right) \int_{t_{0}}^{t_{1}} p(t) \Delta t\right. \\
& \left.-\frac{x^{\sigma}\left(t_{0}^{+}\right)}{a_{1}^{(n-1)}} \int_{t_{1}}^{t_{2}} p(t) \Delta t-\frac{x^{\sigma}\left(t_{0}^{+}\right)}{a_{2}^{(n-1)} a_{1}^{(n-1)}} \int_{t_{2}}^{t_{3}} p(t) \Delta t\right] .
\end{aligned}
$$

By induction we have,for any natural number $m \geq 2$,

$$
\begin{aligned}
& x^{\Delta^{n-1}}\left(t_{m+1}\right) \leq a_{1}^{(n-1)} a_{2}^{(n-1)} \cdots a_{m}^{(n-1)}\left\{x^{\Delta^{n-1}}\left(t_{0}^{+}\right)\right. \\
& -x^{\sigma}\left(t_{0}^{+}\right)\left[\int_{t_{0}}^{t_{1}} p(t) \Delta t+\frac{1}{a_{1}^{(n-1)}} \int_{t_{1}}^{t_{2}} p(t) \Delta t\right. \\
& +\frac{1}{a_{1}^{(n-1)} a_{2}^{(n-1)}} \int_{t_{2}}^{t_{3}} p(t) \Delta t+\cdots \\
& \left.\left.+\frac{1}{a_{1}^{(n-1)} a_{2}^{(n-1)} \cdots a_{m}^{(n-1)}} \int_{t_{m}}^{t_{m+1}} p(t) \Delta t\right]\right\} . \\
& \leq a_{1}^{(n-1)} a_{2}^{(n-1)} \cdots a_{m}^{(n-1)}\left\{x^{\Delta^{n-1}}\left(t_{0}^{+}\right)\right. \\
& \left.-x^{\sigma}\left(t_{0}^{+}\right)\left[\int_{t_{0}}^{t_{m+1}} \prod_{t_{0}<t_{k}<t} \frac{1}{a_{k}^{(n-1)}} p(t) \Delta t\right]\right\} .
\end{aligned}
$$

By (15), (21) and $a_{k}^{(i)}>0$, for all sufficiently lager $m$, we have

$$
x^{\Lambda^{n-1}}\left(t_{m+1}\right)<0 .
$$

This contradicts $x^{\Delta^{n-1}}(t)>0$, for $t \geq T^{\prime}>0$. Hence, every solution of (1) is oscillatory. The proof of theorem 1 is completed.

Corollary 1. Assume the conditions $\left(H_{1}\right)$ holds, and there exists a positive integer $k_{0}$ such that $a_{k}^{(n-1)} \leq 1$ for $k \geq k_{0}$. If $\int^{+\infty} p(t) \Delta t=+\infty$, then every solution of (1) is oscillatory.

Proof. Without loss of generality, let $k_{0}=1$. By $a_{k}^{(n-1)} \leq 1$, we get $\frac{1}{a_{k}^{(n-1)}} \geq 1$, therefore

$$
\int_{t_{0}}^{t} \prod_{t_{0}<t_{k}<s} \frac{1}{a_{k}^{(n-1)}} p(s) \Delta s \geq \int_{t_{0}}^{t} p(s) \Delta s .
$$

Let $t \rightarrow+\infty, \int^{+\infty} p(t) \Delta t=+\infty$, we get that (15) of Theorem 1 holds. By Theorem 1, we know that every solution of (1) is oscillatory.

Corollary 2. Assume the condition $\left(H_{1}\right)$ holds and there exist a positive integer $k_{0}$ and some positive integer $\alpha>0$, such that $\frac{1}{a_{k}^{(n-1)}} \geq\left(\frac{t_{k+1}}{t_{k}}\right)^{\alpha}$, for $k \geq k_{0}$. Furthermore, assume that $\int^{+\infty} t^{\alpha} p(t) \Delta t=+\infty$, then every solution of (1) is oscillatory. 
Proof. By $\frac{1}{a_{k}^{(n-1)}} \geq\left(\frac{t_{k+1}}{t_{k}}\right)^{\alpha}$, we have

$\int_{t_{0}}^{t_{1}} p(t) \Delta t+\frac{1}{a_{1}^{(n-1)}} \int_{t_{1}}^{t_{2}} p(t) \Delta t+\frac{1}{a_{1}^{(n-1)} a_{2}^{(n-1)}} \int_{t_{2}}^{t_{3}} p(t) \Delta t$

$+\cdots+\frac{1}{a_{1}^{(n-1)} a_{2}^{(n-1)} \cdots a_{m}^{(n-1)}} \int_{t_{m}}^{t_{m+1}} p(t) \Delta t$

$\geq\left(\frac{t_{2}}{t_{1}}\right)^{\alpha} \int_{t_{1}}^{t_{2}} p(t) \Delta t+\left(\frac{t_{3}}{t_{1}}\right)^{\alpha} \int_{t_{2}}^{t_{3}} p(t) \Delta t$

$+\cdots+\left(\frac{t_{m+1}}{t_{1}}\right)^{\alpha} \int_{t_{m}}^{t_{m+1}} p(t) \Delta t$

$\geq \frac{1}{t_{1}^{\alpha}}\left[\int_{t_{1}}^{t_{2}} t_{2}^{\alpha} p(t) \Delta t+\int_{t_{2}}^{t_{3}} t_{3}^{\alpha} p(t) \Delta t+\cdots+\int_{t_{m}}^{t_{m+1}} t_{m+1}^{\alpha} p(t) \Delta t\right]$

$\geq \frac{1}{t_{1}^{\alpha}}\left[\int_{t_{1}}^{t_{2}} t^{\alpha} p(t) \Delta t+\int_{t_{2}}^{t_{3}} t^{\alpha} p(t) \Delta t+\cdots+\int_{t_{m}}^{t_{m+1}} t^{\alpha} p(t) \Delta t\right]$

$\geq \frac{1}{t_{1}^{\alpha}} \int_{t_{1}}^{t_{m+1}} t^{\alpha} p(t) \Delta t$.

Let $m \rightarrow+\infty, \int^{+\infty} t^{\alpha} p(t) \Delta t=+\infty$, we get that (15) of Theorem 1 holds. By Theorem 1, we know that every solution of (1) is oscillatory.

\section{Example}

Example. Consider

$$
\left\{\begin{array}{l}
x^{\Delta^{n}}+\frac{1}{4 t} x^{\sigma}(t)=0, t \geq \frac{1}{2}, t \neq k, k=1,2, \cdots \\
x^{(i)}\left(k^{+}\right)=\frac{k}{k+1} x^{(i)}(k), i=0,1, \cdots, n-1 . \\
x\left(\frac{1}{2}\right)=x_{0}, x^{(i)}\left(\frac{1}{2}\right)=x_{0}^{(i)} \\
x(t)=\phi(t), t \in\left[\frac{1}{2}, 1\right]
\end{array}\right.
$$

where $n$ is even, $a_{k}^{(i)}=\frac{k}{k+1}, \quad i=0,1,2, \cdots, n-1$, $p(t)=\frac{1}{4 t}, t_{k}=k, t_{0}=\frac{1}{2}$. For condition $\left(H_{1}\right)$ when $a_{k}^{(i)}=a_{k}^{(i-1)}=\frac{k}{k+1}$

$$
\int_{t_{0}}^{+\infty} \prod_{t_{0}<t_{k}<t} \frac{a_{k}^{(i)}}{a_{k}^{(i-1)}} \Delta t=\int_{t_{0}}^{+\infty} \Delta t=+\infty .
$$

From the above, the condition $\left(H_{1}\right)$ holds. Let

$$
\begin{gathered}
\alpha=1, \frac{1}{a_{k}^{(n-1)}}=\frac{k+1}{k} \geq \frac{t_{k+1}}{t_{k}}=\frac{k+1}{k}, \\
\int^{+\infty} t^{\alpha} p(t) \Delta t=\int^{+\infty} t p(t) \mathrm{d} t=\int^{+\infty} t \frac{1}{4 t} \Delta t=\int^{+\infty} \frac{1}{4} \Delta t=+\infty .
\end{gathered}
$$

By Corollary 2, we know that every solution of (22) is oscillatory.

\section{REFERENCES}

[1] R. P. Agarwal and M. Bohner, "Basic Calculus on Time Scales and Some of Its Applications,” Results in Mathematics, Vol. 35, 1999, pp. 3-22.

[2] M. Bohner and A. Peterson, "Dynamic Equations on Time Scales: An Introduction with Applications,” Birkhäuser, Boston, 2001.

[3] L. H. Erbe, "Oscillation Criteria for Second Order Linear Equations on Atime Scale," Canadian Applied Mathematics Quarterly, Vol. 9, No. 4, 2001, pp. 345-375.

[4] A. Del Medico and Q. K. Kong, "Kamenev-Type and Interval Oscillation Critera for Second-Order Linear Differential Equations on a Measure Chain," Journal of Mathematical Analysis and Applications, Vol. 294, 2004, pp. 621-643. doi:10.1016/j.jmaa.2004.02.040

[5] M. Benchohra, S. Hamani and J. Henderson, "Oscillation and Nonoscillation for Impulsive Dynamic Equations on Certain Time Scales,” Advances in Difference Equations, Vol. 2006, 2006, pp. 1-12. doi:10.1155/ADE/2006/60860

[6] M. Benchohra, J. Henderson, S. K. Ntouyas and A. Ouahab, "On First Order Impulsive Dynamic Equations on Time Scales,” Journal of Difference Equations and Applications, Vol. 10, No. 6, 2004, pp. 541-548. doi:10.1080/10236190410001667986

[7] L. Erbe and A. Peterson, "Oscillation Criteria for Second-Order Matrix Dynamic Equations on Time Scales," Journal of Mathematical Analysis and Applications, Vol. 275, 2002, pp. 418-438. doi:10.1016/S0022-247X(02)00390-6 